Original article

\title{
Evaluation of the spiritual health of cancer patients and their nurses in Iran
}

\author{
Nader Aghakhani ${ }^{1}$, Masoumeh Akbari ${ }^{2 *}$, Mohammad Abbasi ${ }^{3}$, Jaleh Naderi ${ }^{4}$, Rozita Cheraghi ${ }^{5}$, Mohammad \\ Ayremloo $^{6}$, Zoleikha Asgarlou ${ }^{7}$
}

(Received: 4 Mar 2016; Accepted: 16 May 2016)

\begin{abstract}
Background and Purpose: Cancer is associated with significant changes in the lifestyle of patients. Spiritual well-being has been shown to be an inherent element of health, quality of life, increased survival rate, and enhanced adaptability of cancer patients with the disease. This study aimed to evaluate the spiritual health of cancer patients and their nurses.

Methods: This descriptive, correlational, cross-sectional study was conducted on 60 hospitalized cancer patients and 60 nurses in the hospitals affiliated to Urmia University of Medical Sciences, Iran during March-October 2014. Participants were selected via accident sampling, and data were collected using the Spiritual Well-being Scale (SWBS). Data analysis was performed in SPSS version 16 using descriptive statistics, Chi-square and T-test.

Results: In cancer patients, mean scores of religious, existential and spiritual well-being were 52 $\pm 2.32,46 \pm 1.23$ and $99 \pm 3.73$, respectively. In the studied nurses, these scores were $29 \pm 4.43,40 \pm 1.17$ and $70 \pm 4.02$, respectively. A significant difference was observed between the scores of spiritual well-being between cancer patients and nurses $(P=0.001)$.

Conclusion: According to the results of this study, spiritual well-being largely contributes to the mental health of cancer patients. Therefore, higher spiritual well-being of nurses could enhance the spiritual health of cancer patients as well.
\end{abstract}

Keywords: Cancer patients, Nurses, Spiritual health

\section{Introduction}

According to the estimation of the World Health Organization (WHO) in 2012, 14.1 million new cancer cases are reported annually, and 1.7 million cancer deaths occur in Europe each year. Diagnosis of cancer is often unexpected, and ability of the patients to adapt to this condition is of paramount importance. Moreover, access to adequate care and support is crucial for cancer patients (1-3).

In Iran, four million newly diagnosed cancer cases and 8.2 cancer deaths have been reported (4). Since cancer diagnosis causes tremendous stress, disclosure of cancer diagnosis and the relevant information to the patients and their families is challenging. Patients with critical conditions such as cancer need special demands, and many of them resort to spirituality. These patients find spiritual adjustment to be the most effective approach to cope with their disease (5).

\footnotetext{
${ }^{1}$ Inpatient Safety Research Center, Urmia University of Medical Sciences, Urmia, Iran

2,* Corresponding author: Department of Nursing, Faculty of Nursing \& Midwifery, Qom University of Medical Sciences, Qom, Iran. Email:m.akbari43@gmail.com

${ }^{3}$ Department of Nursing, Faculty of Nursing \& Midwifery, Qom University of Medical Sciences, Qom, Iran

${ }^{4}$ Department of Reproductive Health, Pune University, India

${ }^{5}$ Department of Nursing, Urmia University of Medical Sciences, Urmia, Iran

${ }^{6}$ Education Vice Chancellor, Urmia University of Medical Sciences, Urmia, Iran

${ }^{7}$ Department of Midwifery, Zanjan University of Medical Sciences, Zanjan, Iran
} 
Cancer patients may experience low self-esteem and spiritual faith, as well as lack of confidence and inability to use former adaptive mechanisms, which lead to a sense of loneliness, giving rise to a spiritual crisis in the patients. Spiritual crisis is associated with mental and physical imbalance in the life of an individual (6).

Spiritual well-being is highly effective in the proper management of chronic diseases. Meeting the emotional and spiritual needs of chronic patients is a key element of holistic nursing care. Effective post-diagnosis psychosocial adaptive approaches should involve the provision of care in accordance with the physical, psychological, social, and ethical background of patients, as well as their tendencies toward faith, religious beliefs and spiritual perceptions. Nevertheless, most cancer patients do not receive the required spiritual care from their caregivers (4-6). Risk of depression has been reported to be higher in cancer patients whose emotional and spiritual needs are unmet (6). Therefore, cancer patients must be provided with physical, mental, social and spiritual care immediately after disease diagnosis (7).

According to the literature, regular spiritual care increases the life expectancy of chronic patients (8). Patients with psycho-spiritual well-being have a higher capability to cope with the process of terminal diseases (9). As proposed by numerous researchers, spiritual well-being and religious inclinations have a positive impact on the quality of care (10). If spiritual distress is diminished through the provision of spiritual care, it is easier for chronic patients to cope with their disease and end-of-life stages (6).

Due to constant interaction with cancer patients to offer health services, healthcare professionals and nurses are responsible for meeting the psychosocial, emotional, and spiritual needs of cancer patients (7). In this regard, the main challenge for nurses lies in meeting the mental, social, cultural, spiritual, and developmental needs of cancer patients (8). In reality, the nurses should prepare to give spiritual care and respond to patients' spiritual needs (9).

Several studies have assessed the spiritual health of cancer patients and nurses across the world. However, limited research is available on the spiritual health of cancer patients and their caregivers in Iran. This study aimed to evaluate the spiritual well-being of cancer patients and their nurses in order to clarify whether nurses with high spiritual health are able to provide more effective care for these patients.

\section{Materials and Methods}

This cross-sectional, correlational study was conducted on 60 hospitalized cancer patients and 60 nurses at two teaching hospitals affiliated to Urmia University of Medical Sciences, Iran during MarchOctober 2014. Study protocol was approved by the Ethics Committee of Urmia University of Medical Sciences, and required permits were obtained from related authorities prior to data collection. Moreover, written informed consent was obtained from all the subjects prior to the study, and participation in the research was voluntary. Selected cancer patients and nurses were assured of confidentiality terms regarding their personal information.

Demographic characteristics of cancer patients were collected using questionnaires including data on age, disease duration, education status, occupational status, medical history, awareness of the disease, and perspective of patients toward their future and life expectancy. As for nurses, data were provided on variables of age and work experience.

Data collection instrument was the standard Iranian version of Spiritual Well-being Scale (SWBS) (Paloutzian \& Ellison) consisting of two subscales of religious well-being (RWB) (10 items) and existential well-being (EWB) (10 items). Moreover, SWBS is used to assess different mental aspects of individuals, such as the philosophy of life, perceptions of life goals, love and forgiveness.

Validity of the demographic questionnaire was confirmed by the faculty members of Urmia University of Medical Sciences and Islamic Azad University (Urmia branch, Iran). It is noteworthy that SWBS has been applied in previous studies in Iran. Reliability of this scale has been determined using the Cronbach's alpha in a pilot study performed on 20 cancer patients. The validity of this scale has been used by content validity, and its reliability was determined at Cronbach's alpha of 0.82 (11).

Data analysis was performed in SPSS version 
16 using descriptive statistics (mean, frequency, percentage, and standard deviation). In addition, independent sample T-test was used to compare the mean scores of demographic characteristics, and Pearson's correlation-coefficient was used to determine the associations between demographic characteristics and SWBS scores. In this study, P value of less than 0.05 was considered statistically significant.

\section{Results}

Mean age of cancer patients was $46 \pm 17.0$ years (age range: 15-89 years). Among the studied patients, $60 \%$ were literate, and $88 \%$ were self-employed or housewives. Duration of disease in $50 \%$ of the patients was less than one year, $75 \%$ had no history of cancer, and $75 \%$ were aware of their disease. Moreover, $70 \%$ of the patients and $71 \%$ of their families had a positive perception of the disease and life expectancy of the patients.

In this study, mean age of the nurses was $27 \pm 4.6$ years (age range: 23-46 years). According to our findings, $83 \%$ of the studied nurses had work experience of 1-5 years. Among the nurses, mean score of RWB was $29 \pm 4.43$, while the mean score of EWB was $40 \pm 1.17$ and mean score of spiritual health was $70 \pm 4.02$ ( $95 \%$ confidence interval).

Obtained results of this study were indicative of significant differences in the scores of RWB and spiritual health of cancer patients. Moreover, significant differences were observed in cancer patients in terms of RWB, spiritual health, awareness of the disease, and their perspective toward the future and life expectancy. Differences between the RWB scores of cancer patients and perception of their families toward the disease are shown in Table 1.

Our findings were suggestive of significant differences in the EWB and spiritual health of the studied nurses. Furthermore, significant differences were observed between the age and work experience of oncology ward nurses and scores of RWB and spiritual health.

In this study, we determined the mean scores of spiritual health for nurses, and EWB of nurses was found to be higher compared to their RWB. In general, scores of RWB and spiritual health of cancer patients were higher compared to the studied nurses (Table 2).

\section{Discussion}

According to the literature, spiritual well-being plays a pivotal role in preventing diseases and improving the health of patients with physical and psychological problems. In the present study, spiritual well-being of cancer patients was observed to be at a moderate level, which is in line with previous studies in this regard. According to our findings, spiritual well-being of cancer patients was moderately correlated with psychological and spiritual adjustment. Furthermore, several studies have suggested that religious and spiritual wellbeing could effectively promote the optimism of

Table 1. Awareness of patients regarding their disease, perspective of families toward cancer, and religious, existential and spiritual health of cancer patients $(n=60)$

\begin{tabular}{lccc}
\hline Well-being Characteristics & Religious well-being & Existential well-being & Spiritual health \\
\hline Patient's awareness of cancer & 0.001 & 0.001 & 0.001 \\
Patient's perspective toward future and life expectancy & 0.016 & 0.025 & 0.001 \\
Family's perspective toward cancer & 0.085 & 0.001 \\
\hline
\end{tabular}

Table 2. Comparison of spiritual health between cancer patients and oncology ward nurses

\begin{tabular}{lcccc}
\hline \multirow{2}{*}{ Well-being } & \multicolumn{2}{c}{ Cancer patients (n=60) } & \multicolumn{2}{c}{ Nurses (n=60) } \\
\cline { 2 - 4 } Rean & Standard deviation & Mean & Standard deviation \\
Religious well-being & $52(41-60)$ & 2 & $29(21-46)$ & 4 \\
Existential well-being & $46(28-60)$ & 7 & $40(32-52)$ & 4.043 \\
Spiritual health & $99(75-120)$ & 10 & $70(59-83)$ & 4 \\
\hline
\end{tabular}


cancer patients (12-19).

In a study, Baljani (2012) stated that cancer patients experience feelings of hopelessness, loneliness and depression, failing to adapt to their condition. Therefore, it was recommended that nurses and physicians pay special attention to the religious and spiritual well-being of these patients in order to promote their hopefulness (1).

Most of the participants in the current study had a favorable perception of their prognosis, which positively influenced the process of care and enhanced their spiritual well-being. This finding is in congruence with the results obtained by Seyedrasooly (20).

On the other hand, the study by Aghahoseini (2012) revealed that awareness of the disease had no significant effect on the spiritual well-being of cancer patients (11). This is inconsistent with our findings since we observed a significant difference between the RWB, EWB and spiritual health of oncology ward nurses.

According to the study by Musarezaie (2013), nurses are the first caregivers to recognize the spiritual needs of patients and their families, and this recognition must come into effect in meeting the spiritual needs of chronic patients and their relatives. Therefore, it is strongly recommended that a holistic care plan be done into spiritual care promotion of cancer patients in order to enhance their knowledge through relevant training and education (13). Various studies have highlighted the key role of nurses in promoting the spiritual wellbeing of chronic patients $(7,17,20)$. According to a research by Vallurupalli (2012), physicians (87\%) and nurses (85\%) were significantly involved in the enhancement of spiritual well-being among cancer patients (21).

In this regard, findings of Peteet (2013) confirmed the remarkable effect of religious and spiritual well-being on quality of life and decision-making of cancer patients. Furthermore, it was stated that development of spirituality could lead to improved spiritual well-being and avoidance of invasive procedures at end of life stages (20).

In another study by $\mathrm{Oh}$, spiritual intervention was reported to have a significant, moderate impact on the spiritual well-being, life perception, and depression of patients (22). In this regard, Hong (2015) denoted social support and self-esteem to remarkably influence $68 \%$ of the studied patients, emphasizing on the importance of social support in cancer patients (23). Results of several studies have confirmed a positive correlation between the spiritual well-being and overall health of different patients (24).

\section{Ethical considerations}

This study was approved by the Ethics Committee of Islamic Azad University of Urmia branch, Urmia, Iran. Objectives of the study were explained to all the subjects, and written informed consent was obtained from all the samples prior to participation. In addition, participants were assured of confidentiality terms regarding their personal information and were allowed to withdraw from the study at any time.

\section{Study limitations}

The main limitation of the present study was probably the application of our findings. Furthermore, lack of specific diagnostic techniques and genetic testing was another limitation, which might have led to inaccurate prognosis and influenced the perception of cancer patients toward prognosis, as well as their mental state. Consequently, evaluation of the role of cultural, religious and ethnic factors was not possible, and the results may not be generalized to other cultural populations in Iran. Therefore, it is recommended that similar studies be carried out on individuals with different diagnosis and ethnic backgrounds in order to compare the perceptions of chronic patients toward the actual prognosis of the disease.

\section{Conclusion}

According to the results of this study, spiritual wellbeing plays a pivotal role in improving the health of chronic patients. Furthermore, spiritual well-being increases the life expectancy of cancer patients and prevents mental complications such as distress, anxiety, helplessness, hopelessness and fear of death. 
Therefore, it is recommended that relevant training be implemented for oncology ward nurses in order to promote their knowledge and skills regarding the complexity of spiritual care for cancer patients.

\section{Conflicts of interest}

The authors have no conflicts of interest.

\section{Authors' contributions}

N. Aghakhani, M. Akbari, M. Abbasi, J. Naderi, and Z. Asgarlou was responsible for the study concept, drafting of the original manuscript, data acquisition and sampling; M. Akbari analyzed the data; N. Aghakhani and M. Akbari supervised the study. All authors contributed equally to the drafting of the manuscript and critical revision of the paper. The final version of the manuscript was reviewed and confirmed by all the authors before submission.

\section{Acknowledgments}

This article was extracted from a research project approved by the Inpatient Safety Research Center of Urmia University of Medical Sciences and the Islamic Azad University of Urmia branch (Urmia, Iran). Hereby, we extend our gratitude to all the nurses and patients for assisting us in this study.

\section{References}

1. Baljani E, Khashabi J, Amanpour E, Azimi N. Relationship between spiritual well-being, religion, and hope among patients with cancer. J Hayat 2011; 17(3):27-37 (Persian).

2. Molassiotis A, Uyterlinde W, Hollen PJ, Sarna L, Palmer P, Krishnasamy M. Supportive care in lung cancer: milestones over the past 40 years. J Thorac Oncol 2015; 10(1):10-8.

3. Bernard WS, Christopher PW. World cancer report 2014. Geneva: World Health Organization; 2014.

4. Hatamipour K, Rassouli M, Yaghmaie F, Zendedel K, Majd HA. Spiritual needs of cancer patients: a qualitative study. Indian J Palliat Care 2015; 21(1):61-7.

5. Heidarzadeh M, Rassouli M, Shahbolaghi FM, Majd HA, Karam AM, Mirzaee H, et al. Posttraumatic growth and its dimensions in patients with cancer. Middle East J Cancer 2014; 5(1):23-9.

6. Pearce MJ, Coan AD, Herndon JE 2nd, Koenig HG,
Abernethy AP. Unmet spiritual care needs impact emotional and spiritual well-being in advanced cancer patients. Support Care Cancer 2012; 20(10):2269-76.

7. Phelps AC, Lauderdale KE, Alcorn S, Dillinger J, Balboni MT, Van Wert M, et al. Addressing spirituality wellbeing within the care of patients at the end of life: perspectives of patients with advanced cancer, oncologists, and oncology nurses. J Clin Oncol 2012; 30(20):2538-44.

8. Puchalski CM. The role of spirituality wellbeing in health care. Proc (Bayl Univ Med Cent) 2001; 14(4):352-7.

9. Lin HR, Bauer-Wu SM. Psycho-spiritual well-being in patients with advanced cancer: an integrative review of the literature. J Adv Nurse 2003; 44(1):69-80.

10. McNeil SB. Spirituality in adolescents and young adults with cancer a review of literature. J Pediatr Oncol Nurs 2016; 33(1):55-63.

11. Sadat Aghahosseini S, Rahmani A, Abdollahzadeh F, Asvadi Kermani I. The relation between awareness of cancer diagnosis and spiritual health among cancer patients. J Caring Sci .2012; 1(2):67-72.

12. McCaughan E, Parahoo K. Medical and surgical nurses' perceptions of their level of competence and educational needs in caring for patients with cancer. J Clin Nurs 2000; 9(3):420-8.

13. Frost $\mathrm{MH}$, Brueggen $\mathrm{C}$, Mangan $\mathrm{M}$. Intervening with the psychosocial needs of patients and families: perceived importance and skill level. Cancer Nurs 1997; 20(5):350-8.

14. Chan MF. Erratum. Factors affecting nursing staff in practicing spiritual care. J Clin Nurs . 2015; 24(17-18):2684.

15. Mahmoodishan G, Alhani F, Ahmadi F, Kazemnejad A. Iranian nurses' perception of spirituality and spiritual care: a qualitative content analysis study. J Med Ethics Hist Med 2010; 3:6.

16. Paloutzian RF, Park CL. Handbook of the psychology of religion and spirituality. $1^{\text {st }}$ ed. New York: Guilford Publication; 2014.

17. Li CC, Rew L, Hwang SL. The relationship between spiritual well-being and psychosocial adjustment in Taiwanese patients with colorectal cancer and a colostomy. J Wound Ostomy Continence Nurs 2012; 39(2):161-9.

18. Musarezaie A, Naji-Esfahani H, Momeni-Ghale Ghasemi T, Karimian J, Ebrahimi A. Investigation of the SWB and its relation with demographic parameters in patients with breast cancer referred to an oncology hospital affiliated to the Isfahan University of Medical Sciences. J Educ Health Promot 2013; 2:42.

19. Seyedrasooly A, Rahmani A, Zamanzadeh V, Aliashrafi Z, Nikanfar AR, Jasemi M. Association between perception of prognosis and spiritual well-being among cancer patients. J Caring Sci 2014;3(1):47-55.

20. Peteet JR, Balboni MJ. Spirituality wellbeing and religion in oncology. CA Cancer J Clin 2013; 63(4):280-9.

21. Vallurupalli M, Lauderdale K, Balboni MJ, Phelps AC, 
Block SD, Ng AK, et al. The role of spirituality wellbeing and religious coping in the quality of life of patients with advanced cancer receiving palliative radiation therapy. J Support Oncol. 2012; 10(2):81-7.

22. Oh PJ, Kim SH. The effects of spiritual interventions in patients with cancer: a meta-analysis. Oncol Nurs Forum
2014; 41(5):E290-301.

23. Hong SS, Park HR. Predictors of meaning in life in adolescents with leukemia. Child Health Nurs Res 2015; 21(1):74-81.

24. Visser A, Garssen B, Vingerhoets A. Spirituality and wellbeing in cancer patients: a review. Psycho oncology 2010; 19(6):565-72. 